\title{
Evaluation of Nurse Preferences Between the Lanreotide Autogel New Syringe and the Octreotide Long-Acting Release Syringe: An International Simulated-Use Study (PRESTO)
}

\author{
Daphne Adelman (D) X Xuan-Mai Truong Thanh · Marion Feuilly • \\ Aude Houchard · David Cella
}

Received: January 22, 2020 / Published online: March 11, 2020

(C) The Author(s) 2020

\begin{abstract}
Introduction: Somatostatin analogues are used to treat symptoms and slow tumour progression in patients with neuroendocrine tumours (NETs) and carcinoid syndrome and to reduce hormone secretion and pituitary tumour
\end{abstract}

Enhanced Digital Features To view enhanced digital features for this article, including a summary slide and graphical abstract, go to https://doi.org/10.6084/m9. figshare. 11806605 .

Electronic Supplementary Material The online version of this article (https://doi.org/10.1007/s12325020-01255-8) contains supplementary material, which is available to authorized users.

\section{Adelman ( $\square)$}

Division of Endocrinology, Metabolism and Molecular Medicine, Northwestern University

Feinberg School of Medicine, Chicago, IL, USA

e-mail: d-adelman@northwestern.edu

X.-M. Truong Thanh

Global Medical Affairs, Ipsen, Boulogne-Billancourt, France

M. Feuilly

Health Economics and Outcomes Research, Ipsen,

Boulogne-Billancourt, France

A. Houchard

Biometry, Ipsen, Boulogne-Billancourt, France

D. Cella

Department of Medical Social Sciences,

Northwestern University Feinberg School of

Medicine, Chicago, IL, USA volume in patients with acromegaly. A new syringe for lanreotide autogel/depot (LAN) was developed following feedback from a human factors study to improve ease of injection compared with previous syringes. PRESTO aimed to assess preferences of nurses between the LAN new syringe and the octreotide long-acting release (LAR) syringe.

Methods: PRESTO, a multinational, multicentre, prospective, noninterventional, simulateduse study, enrolled nurses with $\geq 2$ years' experience injecting LAN and/or octreotide LAR in patients with NETs and/or acromegaly. Nurses administered injections into pads using the LAN new syringe and octreotide LAR syringe in a randomised sequence. In an anonymous webbased questionnaire, nurses reported their overall preference ('strong' or 'slight'; primary endpoint) and rated and ranked the importance of nine attributes for each syringe ( 1 [not at all] to 5 [very much]).

Results: Overall, 90 nurses attended sessions and completed valid questionnaires. Most nurses $(97.8 \%)$ expressed a preference $(85.6 \%$ 'strong', $12.2 \%$ 'slight') for the LAN new syringe versus the octreotide LAR syringe $(P<0.0001)$. Attribute performance ratings ( 1 [not at all] to 5 [very much]) were consistently higher for the LAN new syringe versus the octreotide LAR syringe, with the greatest differences in 'fast administration' and 'confidence the syringe will not be clogged' (mean difference [SD]: 2.6 [1.2] and $2.3[1.5], \quad$ respectively; $P<0.0001)$. 
The attribute ranked most important was 'confidence the syringe will not be clogged' (24.4\%); least important was 'convenience of syringe format, including packaging, from preparation to injection' (34.4\%).

Conclusions: Nurses preferred the user experience of the LAN new syringe compared with the octreotide LAR syringe, with a particular preference for attributes related to product delivery with the LAN new syringe.

Keywords: New syringe; Nurse preference; Simulated injection; Somatostatin analogue

\section{Key Summary Points}

\section{Why Carry out This Study?}

The lanreotide autogel/depot (LAN) new syringe was developed in collaboration with patients, caregivers and healthcare professionals to ensure it met their needs, and a validation study has confirmed that it can be used effectively and safely.

Human factors engineering represents a new sector of syringe designing as well as the recognised need to focus on improving the injection experience by engaging with those who use the syringes during the design process.

To test a new delivery system, it should be assessed in an environment that simulates a real injection.

The LAN new syringe is now approved for use in several countries. The aim of the present study (PRESTO) was to assess the preferences of nurses between the LAN new syringe and the current octreotide long-acting release (LAR) syringe after performing simulated injections.

\section{What Was Learned From the Study?}

In this study, almost all nurses (97.8\%) reported a preference for the LAN new prefilled/ready-to-use syringe compared with the current octreotide LAR syringe.
The syringe attribute ranked most important to nurses was 'confidence that the syringe will not be clogged'.

The results of the PRESTO study suggest that the LAN new syringe may improve user experience compared with existing products when used in clinical practice.

\section{INTRODUCTION}

Somatostatin analogues (SSAs) are used to treat symptoms and slow tumour progression in patients with neuroendocrine tumours (NETs) and carcinoid syndrome and to reduce hormone secretion and reduce pituitary tumour volume in patients with acromegaly [1-5]. NETs, carcinoid syndrome and acromegaly are chronic conditions requiring long-term treatment [6], so it is important to optimise patients' treatment experience. One way to improve patient experience is through refinement of the drug delivery system.

For long-acting SSAs, nurses identified usability, convenience and safety as key factors in determining healthcare professional experience $[7,8]$. In a previous preference study published in 2012, nurses preferred the lanreotide autogel/depot (LAN) syringe compared with that of previous octreotide long-acting release (LAR) $[7,8]$. Although nurses preferred the LAN syringe overall and for nearly all syringe attributes, the sturdiness of the plunger received a higher rating for the octreotide LAR syringe [7]; this is consistent with feedback from patients, caregivers and healthcare professionals obtained during a series of formative interviewbased studies [9].

Based on these previous findings, and to continue to improve patient and nurse experience, the LAN new syringe was developed to be more ergonomic and user-friendly than the previous delivery system [9]. The key changes made to this device included larger flanges (finger supports for pushing the plunger down), new plunger supports, a larger, ridged, non- 
transparent needle cap (previously a seethrough needle shield) and a new protective tray [9]. The present study is the first to ask experienced nurses to report their preference between the LAN new syringe and the current octreotide LAR syringe.

\section{METHODS}

\section{Study Design}

The PREference STudy of lanreOtide autogel (PRESTO) was a multinational, multicentre, noninterventional, simulated-use study involving nurses with experience managing patients with NETs and/or acromegaly by injecting longacting SSAs. This study was performed at clinical or market research centres in countries where the LAN new syringe was approved prior to or during the testing period (if during, testing sessions were initiated after the approval date): Belgium, Germany, Italy, Spain, the UK and the USA. All centres allowed for the shipment of study drug to these locations and storage in refrigerators.

Nurses attended a single testing session between June 2019 and September 2019, during which they injected injection pads (Remedy Simulation Group, Perkasie, PA, USA, or 3B Scientific, Somerset, UK) with each syringe twice (randomised injection order) before reporting their preferences. Data were collected using an anonymous, self-administered, cross-sectional, web-based questionnaire on a tablet device.

\section{Recruitment and Eligibility Criteria}

Nurses from organisations and institutions where patients with acromegaly and/or NETs were routinely treated were targeted for recruitment. Identified nurses were sent an invitation email, which contained a nurse identification form to assess eligibility and qualifications. Further details of the recruitment process are available in the supplementary materials.

Eligible participants were adults aged $\geq 18$ years, employed as a hospital- or community-based nurse, or nurse from a local physician's office or nurses' network, with $\geq 2$ years' current and/or previous experience administering long-acting SSA injections to patients with acromegaly and/or NETs $(\geq 4$ acromegaly and/or NETs patients per year; or $\geq 1$ every 3 months). No specific exclusion criteria were defined.

All enrolled nurses signed a questionnaire participation consent form to participate in testing sessions and to complete the questionnaire.

\section{Testing Sessions}

Eligible nurses were invited to attend a 60-90-min testing session (up to seven nurses per session). Sessions were standardised across testing centres and conducted in the local language (English, French, Flemish, German, Italian or Spanish). At these sessions, clinical research associates (CRAs) provided nurses with injection-related testing resources, including leaflets with information about each syringe and a slide deck that included a study overview, and detailed instructions for testing the syringes and completing the electronic questionnaire.

Nurses were provided with materials to administer four simulated injections (two with each product: LAN $120 \mathrm{mg}$ and octreotide LAR 20 or $30 \mathrm{mg}$ ) into two injection pads (one per product). Both syringes were provided by Ipsen, contained real products and were labelled 'not for human use' on the box in their local language. LAN new syringe packs consisted of a pre-filled, ready-to-use syringe $(0.5 \mathrm{ml}$ with an automatic safety system and a $1.2 \times 20 \mathrm{~mm}$ needle) in a laminated pouch. Octreotide LAR syringe packs contained: one 6-ml glass vial with a rubber stopper, sealed with an aluminium flip-off seal, containing powder for suspension for injection; one $3 \mathrm{ml}$ pre-filled glass syringe with a front and plunger stopper with $2 \mathrm{ml}$ solvent, co-packaged in a sealed blister tray; one vial adapter; one safety injection needle. Before each session, products were retrieved from the refrigerator at least $30 \mathrm{~min}$ prior to the testing as per instructions for use on the label $[10,11]$.

The order of injections with each product was randomised using a pre-populated 
randomisation list with block sizes of two to ensure counter balance in the ordering of syringes throughout each session and the study. CRAs at each session were given a unique section of the randomisation list to prepopulate the session $\log$, and nurses were assigned a randomisation number consecutively upon arrival.

After testing each syringe twice, nurses completed the web-based questionnaire using a tablet device. CRAs completed the session and randomisation number fields for each participant. Once the questionnaire was completed, data were saved on a secure central server.

\section{Objectives and Assessments}

To develop the current web-based questionnaire, a systematic literature review, detailed in the supplementary materials, was performed to identify relevant previous preference studies that utilised a questionnaire methodology with additional input from a patient-reported outcomes expert (DC) and nurses (including DA). The web-based questionnaire, comprised of a single four-level preference question, and the rating and ranking of nine pre-defined attributes, is provided in full in the supplementary materials.

The primary objective of PRESTO was to assess nurses' preference for the LAN new syringe compared with the octreotide LAR syringe after performing injections into injection pads. This was assessed by the proportion of nurses who stated a strong/slight preference for each syringe in response to an overall preference item with four response options (slight or strong preference for the LAN new syringe or the octreotide LAR syringe).

Secondary objectives were:

1. To describe nurse preferences for the attributes of each syringe, evaluated through rating nine pre-defined attributes using a 5-point Likert-type response scale from 1 (not at all) to 5 (very much).

2. To describe the importance of syringe attributes. Based on the same nine predefined attributes, nurses were then asked to rank the three most important attributes (in rank order) and one least important attribute. Nurses were also asked if any attributes they considered important were not listed.

3. To describe the clinical characteristics of nurses (e.g., clinical experience, setting).

4. To describe the sociodemographics of nurses (e.g., age, gender).

The exploratory objective was to assess how syringe attributes and factors such as nurse characteristics and sociodemographics related to overall preference.

During nurse sessions, an issue was identified: a number of injections administered using the octreotide LAR syringe resulted in clogging, found to be due to the injection pad, which was consequently changed during the course of the study. Further details on this issue are described in the supplementary materials.

\section{Statistical Analyses}

This study aimed to recruit 90 nurses based on a meaningful difference of approximately 15 percentage points and a preference of $57.5 \%$ for one syringe and $42.5 \%$ for the other syringe. This sample size was based on using a onesample binomial test with a power of $81 \%$ and two-sided 5\% alpha level. This sample size was determined prior to initiating recruitment. Analyses were carried out on the enrolled analysis set (all nurses), consisting of all participants who self-reported meeting all eligibility criteria, as well as by injection pad used, defined as the first pad analysis set (FPAS) and second pad analysis set (SPAS).

The primary outcome was analysed using a one-sample binomial test for proportions of nurses preferring the LAN new syringe compared with the octreotide LAR syringe. The importance of attributes for each syringe are summarised descriptively through frequency of responses, related percentages and the difference in rating between the two syringes using paired $t$ tests. Analysis of syringe attributes was also categorised by scores of $<5$ and 5 (i.e., highest rating, 5 = very much), also known as a 'top-box' score [12-15]. Rankings for each attribute are summarised descriptively through 
frequencies and percentages indicating the first, second and third most important and the least important.

Descriptive analyses were conducted for clinical characteristics and sociodemographics of nurses, including frequencies and percentages for categorical variables.

Exploratory analyses were performed using univariate logistic regression models to identify parameters associated with overall preference using a significance level of $P<0.20$, presented as the odds ratio (OR; 95\% confidence interval $[\mathrm{CI}])$. Collinearity of these selected variables was examined through correlations, ANOVAs, chisquare or Fisher tests, as appropriate. Variables in the univariable logistic regression model with a significance level of $P<0.2$ were considered for inclusion in a multivariable model.

All data were forced responses; thus, there were no missing data in variables captured. All analyses were performed using $\mathrm{SAS}^{\circledR}$ v9.3 or later (SAS Institute, Cary, NC, USA).

\section{Ethics}

As this study was not a clinical trial and no patients were involved, institutional review board approvals were not required. This study followed the recommendations from the International Society for Pharmacoepidemiology, Good Pharmacoepidemiology Practice Guidelines, April 2007. The confidential nature of personal information was maintained and participating nurses provided authorisation for the use and disclosure of personal information (e.g. age, gender, clinical experience) in the study.

\section{RESULTS}

\section{Nurse Characteristics}

In total, 93 nurses participated in the testing sessions and completed the web-based questionnaire, of which 90 questionnaires were validated; three nurses were not included because of a saving error that meant that their responses were not retrievable. A flow diagram of study recruitment is shown in Fig. 1. In total, 24 testing sessions took place; 10 were conducted using PAD1 $(n=45)$ and 14 using PAD2 $(n=45)$. The mean number of nurses per session was 3.8 (95\% CI 3.0, 4.5) overall, and 4.5 (95\% CI 3.4, 5.6) and 3.2 (95\% CI 2.2, 4.2) in the FPAS and SPAS groups, respectively.

Overall, the mean (SD) age of nurses was 44.2 (10.6) years and $88.3 \%$ were female. The largest proportion of nurses was from Italy $(48.9 \%)$, followed by the USA (27.8\%), UK (8.9\%), Germany $(7.8 \%)$, Belgium and Spain (3.3\% each). Further details of sociodemographic characteristics and clinical setting, including clinical department and data for the FPAS and SPAS, are provided in Table $\mathrm{S} 1$. The clinical experience of nurses is shown in Fig. 2, with further details provided in Table $S 2$. The median length of time nurses had experience injecting LAN and

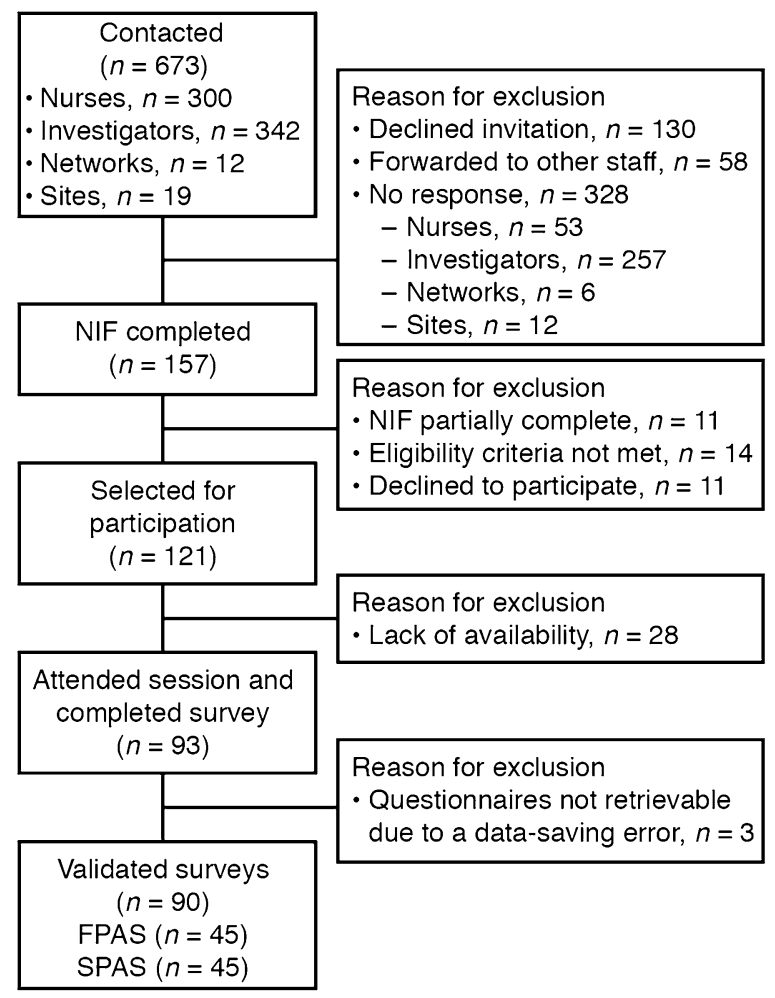

Fig. 1 Recruitment of study nurses. FPAS first pad analysis set, NIF nurse identification form, $S P A S$ second pad analysis set 


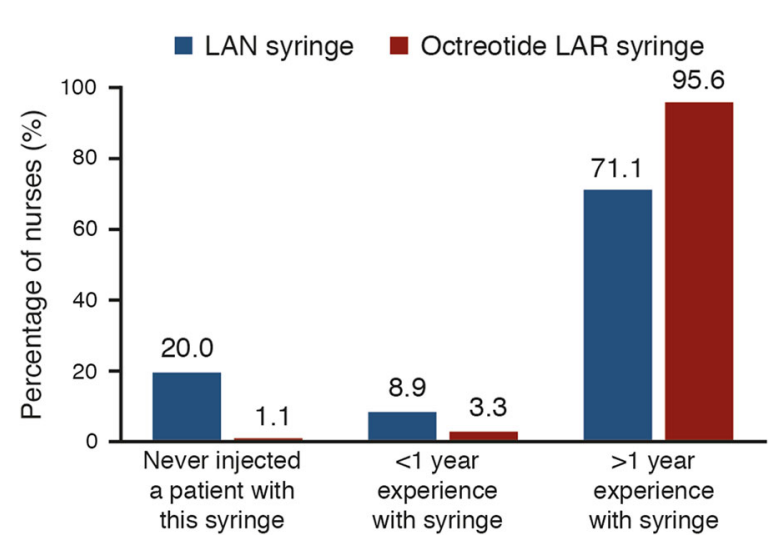

Fig. 2 Clinical experience of nurses, length of time injecting patients using LAN or octreotide LAR. LAN lanreotide, $L A R$ long-acting release. Further details of the clinical experience of nurses are presented in Table S2

octreotide LAR was 4.0 (95\% CI 3.0, 6.0) years and 7.5 (95\% CI 5.0, 10.0) years, respectively.

\section{Overall Preference}

Overall, 97.8\% (95\% CI 92.2, 99.7) of nurses expressed a preference for the LAN new syringe, while $2.2 \%(95 \%$ CI $0.3,7.8)$ preferred the octreotide LAR syringe $(P<0.0001)$. The proportion of nurses who reported a 'slight' or 'strong' preference for each syringe is presented in Fig. 3. The overall reported preferences were the same between the FPAS and SPAS.

\section{Performance Ratings for Syringe Attributes}

Syringe attribute performance ratings for the LAN new syringe and the octreotide LAR syringe are shown in Fig. 4 and Table S3. No notable differences were observed between the FPAS and SPAS; thus, the results described below are for the overall population.

Attribute performance ratings were consistently higher for the LAN new syringe compared with the octreotide LAR syringe, with the greatest differences in ratings for 'fast administration from preparation to injection' and 'confidence that the syringe will not be clogged' (mean difference: 2.6 [95\% CI 2.4, 2.9] and 2.3 $[95 \%$ CI 2.0, 2.6], respectively; $P<0.0001)$.

The proportion of nurses who awarded the highest rating ('very much') in response to the performance statements is presented in Fig. S1. The majority of nurses gave maximum performance ratings for the LAN new syringe on all attributes, ranging from $58.9 \%$ (95\% CI 48.0 , 69.2) for the attribute 'comfortable to hold during use from preparation to injection' to $81.1 \%$ (95\% CI $71.5,88.6)$ for the attribute 'confidence that there is no loss of product during preparation or delivery'. Few nurses gave maximum performance ratings for the octreotide LAR syringe for any attribute.

The attribute most frequently ranked as most important was 'confidence that the syringe will not be clogged' $(24.4 \%$ [95\% CI 16.0, 34.6])

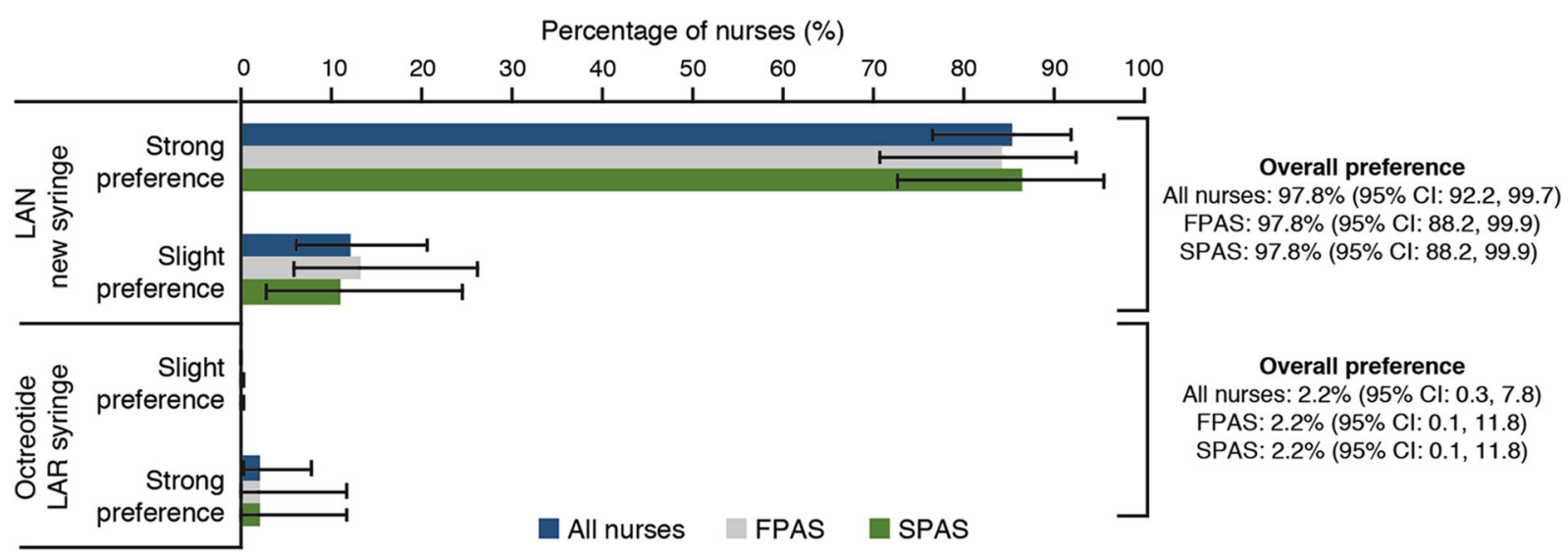

Fig. 3 Reported preferences for somatostatin analogue syringe. Error bars represent the 95\% CIs. CI confidence interval, FPAS first pad analysis set, $L A N$ lanreotide autogel/depot, $L A R$ long-acting release, SPAS second pad analysis set 


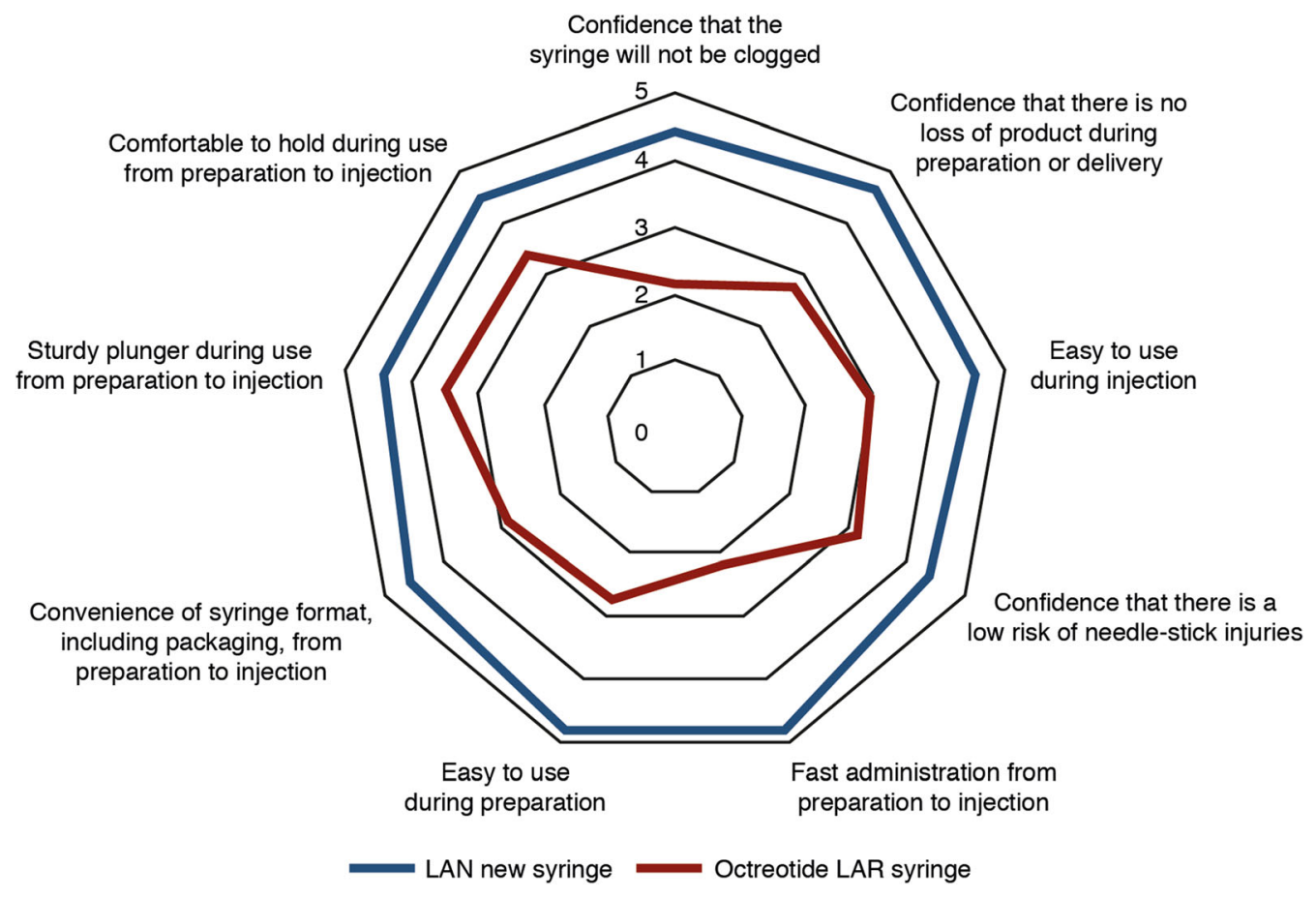

Fig. 4 Evaluation of preference for somatostatin analogue syringe based on the performance of each attribute. Responses in answer to the following survey question: Considering the testing performed earlier today, we would now like you to evaluate the performance from 1 to 5 of LAN new syringe and octreotide LAR syringes on each

followed by 'confidence that there is no loss of product during preparation or delivery' $(21.1 \%$ [95\% CI 13.2, 31.0]), and the least important was 'convenience of syringe format, including packaging, from preparation to injection' $(34.4 \% \quad[95 \%$ CI 24.7, 45.2]) (Fig. 5 and Table S4).

In response to a free-text question, $10.0 \%$ of nurses (FPAS: 11.1\%; SPAS: 8.9\%) considered there to be an important attribute missing from the pre-defined list. The identified missing attributes were related to: patient comfort and safety during and/or after injection, raised by four nurses; biomechanical feedback of the needle/injection during and/or after injection, raised by three nurses; the possibility of selfadministration, raised by two nurses; and the dilution/solubility of octreotide LAR, raised by one nurse. attribute. Please rate each syringe where: $1=$ not at all, $2=$ a little bit, $3=$ somewhat, $4=$ quite a bit, $5=$ very much. Please see Table S3 for SD and 95\% confidence intervals and data for the first and second pad analysis sets. $L A R$ long-acting release

\section{Factors Associated with Overall Preference}

The following variables were associated $(P<0.20)$ with overall preference for the LAN new syringe in a univariate fashion: number of years' experience injecting patients using the octreotide LAR syringe (OR [95\% CI]: 2.2 [0.8, 5.9]), number of patients with NETs and/or acromegaly treated in the last 12 months using octreotide LAR (OR [95\% CI]: $1.5[0.8,2.6])$ and the octreotide LAR syringe attribute performance ratings for 'fast administration from preparation to injection' (OR [95\% CI]: 0.4 [0.1, 1.3]) and 'convenience of syringe format including packaging from preparation to injection' (OR $[95 \% \mathrm{CI}]: 0.2 \quad[0.0,1.4])$. No collinearity was identified, and all pre-selected variables were included into the multivariate model. In the multivariate model, no variables 


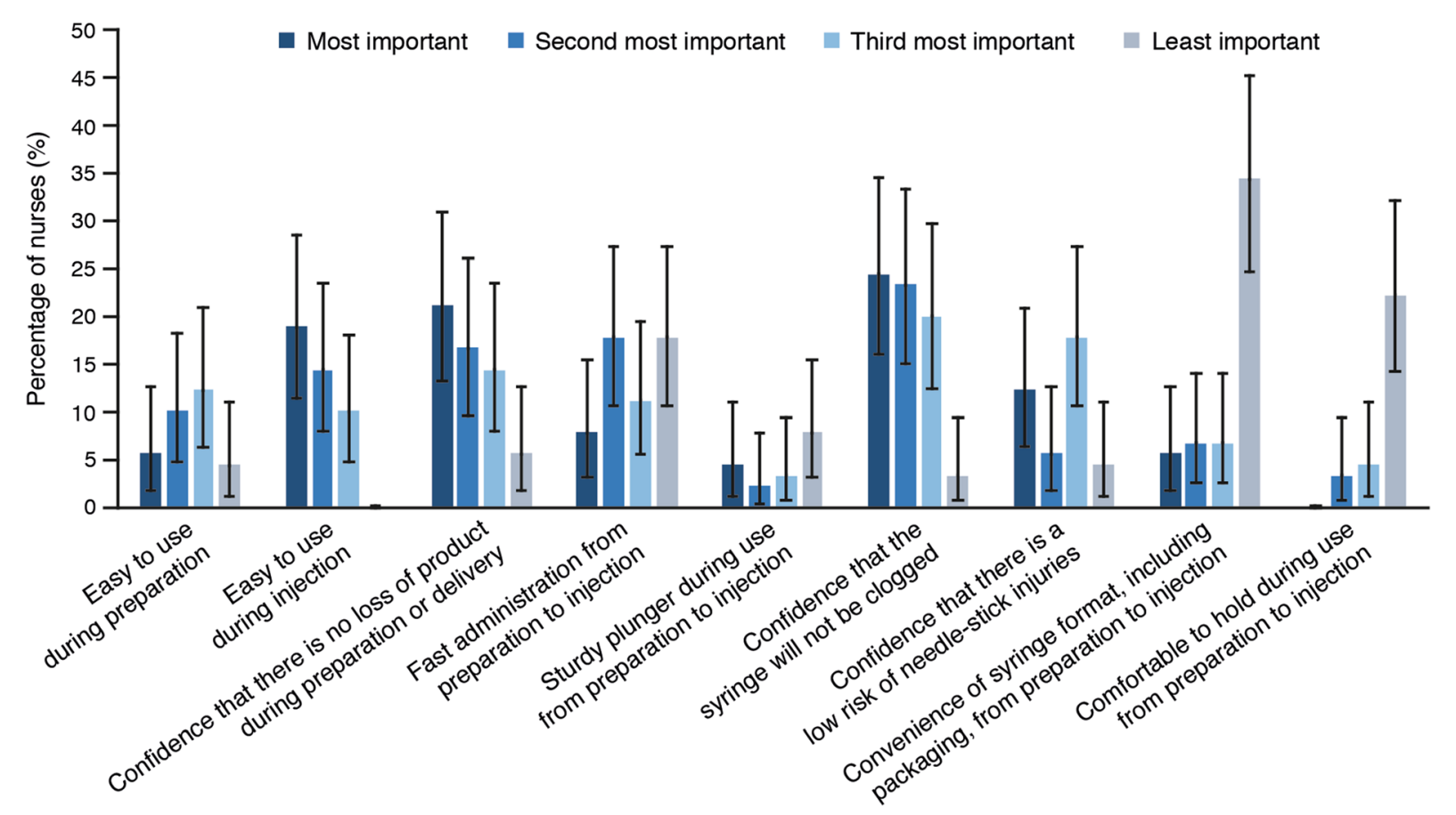

Fig. 5 Ranked importance of syringe attributes according to nurses. Data are presented for all nurses. Error bars represent 95\% confidence intervals. Please see Table S4 for full details and data for the first and second pad analysis sets

were associated with overall preference for the LAN new syringe at the 0.05 level.

\section{DISCUSSION}

In the present study, nurses reported a considerable preference for the LAN new syringe compared with that for the current octreotide LAR syringe; furthermore, when nurses were asked to rate the performance of particular syringe attributes, ratings for each attribute were consistently higher for the LAN new syringe.

Overall, $97.8 \%$ of nurses reported a preference for the LAN new syringe over the octreotide LAR syringe, with the majority of nurses reporting a 'strong' preference. Results were similar for both the FPAS and SPAS for the primary endpoint, suggesting that the initial issue with the octreotide LAR syringe clogging using PAD1 did not impact upon the overall findings. Exploratory analyses also showed that the sociodemographics or clinical experience of nurses did not have an impact on overall preference. In a previous study, the overall preference score by nurse assessment was 63 percentage-points higher for the LAN previous syringe compared with the previous octreotide LAR syringe [7] and, in another study, $81 \%$ of patients who switched from octreotide LAR to LAN preferred to continue treatment with LAN [16]. Given the almost universal preference for the LAN new syringe in the PRESTO study, this suggests that the advancements made for the LAN syringe (larger flanges; a larger, more rigid non-transparent needle cap; new plunger supports; new protective tray) [9] following feedback from nurses [7] and from patients, caregivers and nurses in formative human factors studies [9] resulted in improved user experience with this new device.

Fast administration and confidence that the syringe would not be clogged were the attributes with the greatest differences in performance ratings between the two syringes, in favour of the LAN new syringe, and when ranked in order of importance, confidence that the syringe would not be clogged was the most important attribute. During the study, the issue of clogging with the octreotide LAR syringe was 
found to be caused by the injection pads used; however, no notable difference was observed between the FPAS and SPAS for the performance rating of syringes for this attribute (mean difference in ratings between syringes 2.5 [95\% CI 2.0, 2.9] and 2.1 [95\% CI 1.6, 2.5], respectively) or the percentage of nurses who ranked this as the most important attribute (22.2\% [95\% CI 11.2, 37.1] and 26.7\% [95\% CI $14.6,41.9]$, respectively). This risk of clogging with the previous and current octreotide LAR syringe is not uncommon and has been reported in previous studies, including reporting of nurses and clinical staff in response to questionnaires [7, 17], as well as in studies that report the experience of patients $[18,19]$. It is therefore possible that previous experience may have influenced nurses' responses to this particular attribute.

The second most important attribute identified in the present study was confidence that there would be no loss of product during preparation or delivery, with no difference observed between the percentage of nurses who ranked this attribute as the most important between the FPAS and SPAS $(22.2 \%$ [95\% CI 11.2, 37.1] and 20.0\% [95\% CI 9.6, 34.6]). Nurses have previously identified 'confidence that a full dose has been delivered' as the most important attribute for SSA syringes [7], and it is a concern among patients with chronic diseases who administer self-injections that product can be lost if injections are too shallow [20]. However, in the present study, $81 \%$ of nurses gave the highest rating for the statement regarding confidence of no product was lost with the LAN new syringe compared with $10 \%$ for the octreotide LAR syringe. Furthermore, needle retraction is an established benefit of the LAN previous syringe, contributing to the reported low risk of needlestick injuries compared with the previous octreotide LAR syringe [7]. This feature was carried forward to the LAN new syringe and, as shown in the present study, nurses' 'confidence that there is low risk of needle stick injuries' achieved a mean score of 4.4 (95\% CI $4.2,4.6)$ compared with 3.2 (95\% CI 2.9, 3.4) for the octreotide LAR syringe.

\section{Strengths and Limitations}

The most prominent limitation of this study was the need for a change in injection pad after 10 injection sessions due to clogging issues. This resulted in outcomes being presented for the FPAS and SPAS. It should be noted that injections performed in the present study were simulated and therefore differ from real-life injections in patients and that, since the LAN new syringe was recently launched, there were a limited number of countries where this study could be performed. There were some imbalances noted in sociodemographics and clinical settings of nurses. In the overall population $49 \%$ of nurses were recruited from Italy, followed by $28 \%$ from the USA, and the majority of nurses were recruited from an oncology $(42 \%)$ or endocrinology (43\%) department/ unit/ward. To avoid the global risk of not completing the study within the planned timelines, IQVIA network nurses participated in the study. Consequently, this potentially introduced biased reporting of preferences due to potential conflicts of interest for these nurses. Potential selection bias may also be considered based on nurses' voluntary participation in the study; although perhaps not unusual for this type of study, almost half of nurses invited by email were non-responders and it was considered that only nurses who were willing and felt they met the required criteria may have responded. Altogether, the risk of selection bias was considered minimal, as the nurses did not gain financially from success or failure of the study and all data was anonymised, so they would be likely to state their true preferences.

The strengths of the PRESTO study include the robustness of the study design based on the pre-specified sample size calculation and the randomisation of injection order at the nurse level, which reduced the potential for bias. This study benefited from the recruitment of experienced nurses who perform injections in both NETs and acromegaly patients and who were recruited internationally to avoid a centre or country effect. The questionnaire used in this study was carefully designed through a systematic literature search to identify relevant prior publications and developed with input from the 
end users and a patient-reported outcomes expert. Compared with the previous nurse preference study [7], PRESTO used a choicebased question to assess the nurses' stated preference between syringes, which is a strength of this study, as it is more consistent with reallife decision-making. Improving patients' experience with injectable devices through optimising delivery and gaining user insights on their device experiences and preferences is not unique to treatment with SSAs, but is also a key topic of research in therapy areas such as diabetes [21-23].

\section{CONCLUSION}

The PRESTO study showed that nurses with either past or current experience injecting longacting SSAs in patients with NETs and/or acromegaly in the EU and the USA preferred the user experience of the LAN new syringe over that of the current octreotide LAR syringe, when assessed through simulated injection. The attributes with the greatest difference in scores between syringes were fast administration and confidence the syringe will not be clogged, in favour of the LAN new syringe. These results suggest that the LAN new syringe has overcome the shortcomings identified with the previous LAN syringe and may therefore improve user experience compared with previous and/or current products, when used in clinical practice.

\section{ACKNOWLEDGEMENTS}

The authors thank all nurses who participated in the study. The authors would also like to thank the contract research organisation, IQVIA, for its contribution to study recruitment and conduct of the study sessions; in particular, Mr Paul Williams (Lead Statistician, Real World Solutions, IQVIA), who led the design and scientific aspects, analysis, and interpretation and reporting of results, and Dr Laurie Batchelder (Lead Medical Writer, Real World Solutions, IQVIA), who substantially contributed to the design of the survey and protocol, statistical analysis plan and discussion of the report.
Funding. Sponsorship for this study and the journal's Open Access and Rapid Service fee were funded by Ipsen.

Medical Writing and Editorial Assistance. The authors thank Jacqueline Harte and Helen Marshall of Watermeadow Medical, an Ashfield company, for providing medical writing and editorial support, which was sponsored by Ipsen in accordance with Good Publication Practice guidelines.

Authorship. All named authors meet the International Committee of Medical Journal Editors (ICMJE) criteria for authorship for this article, take responsibility for the integrity of the work as a whole, and have given their approval for this version to be published. All authors contributed to the study conception/ design, or acquisition, analysis, and/or interpretation of data. All authors were involved in drafting and/or critical revision of the manuscript for intellectual content and provided final approval for publication. Ipsen was involved in the study design, conduct, and analysis of this study, and provided funding for the medical writing support to develop this publication. Study recruitment and conduct of the study sessions was managed by the contract research organisation, IQVIA.

Prior Presentation. An abstract has been submitted for presentation at ENETS, Barcelona, Spain, 11-13th March 2020.

Disclosures. DA received: consultancy fees from Pfizer and Novo Nordisk; and has served on an advisory board for Ipsen, Crinetics and Novo Nordisk. DC received: consultancy fees from Ipsen and Novartis; and grant support from Ipsen and Novartis. XMTT, MF and AH are employees of Ipsen.

Compliance with Ethics Guidelines. As this study was not a clinical trial and no patients were involved, institutional review board approvals were not required. This study followed the recommendations from the International Society for Pharmacoepidemiology, Good Pharmacoepidemiology Practice Guidelines, 
April 2007. The confidential nature of personal information was maintained and participating nurses provided authorisation for the use and disclosure of personal information in the study.

Data Availability. Where participant data can be anonymised, Ipsen will share all individual participant data that underlie the results reported in this article with qualified researchers who provide a valid research question. Study documents, such as the study protocol and clinical study report, are not always available. Proposals should be submitted to DataSharing@Ipsen.com and will be assessed by a scientific review board. Data are available beginning 6 months and ending 5 years after publication; after this time, only raw data may be available.

Open Access. This article is licensed under a Creative Commons Attribution-NonCommercial 4.0 International License, which permits any non-commercial use, sharing, adaptation, distribution and reproduction in any medium or format, as long as you give appropriate credit to the original author(s) and the source, provide a link to the Creative Commons licence, and indicate if changes were made. The images or other third party material in this article are included in the article's Creative Commons licence, unless indicated otherwise in a credit line to the material. If material is not included in the article's Creative Commons licence and your intended use is not permitted by statutory regulation or exceeds the permitted use, you will need to obtain permission directly from the copyright holder. To view a copy of this licence, visit http://creativecommons.org/licenses/by$\mathrm{nc} / 4.0 /$.

\section{REFERENCES}

1. Caplin ME, Pavel M, Cwikla JB, et al. Lanreotide in metastatic enteropancreatic neuroendocrine tumors. N Engl J Med. 2014;371:224-33.

2. Rinke A, Muller $\mathrm{HH}$, Schade-Brittinger $\mathrm{C}$, et al. Placebo-controlled, double-blind, prospective, randomized study on the effect of octreotide LAR in the control of tumor growth in patients with metastatic neuroendocrine midgut tumors: a report from the PROMID Study Group. J Clin Oncol. 2009;27:4656-63.

3. Strosberg J, Kvols L. Antiproliferative effect of somatostatin analogs in gastroenteropancreatic neuroendocrine tumors. World J Gastroenterol. 2010;16:2963-70.

4. Caron PJ, Bevan JS, Petersenn S, et al. Tumor shrinkage with lanreotide Autogel $120 \mathrm{mg}$ as primary therapy in acromegaly: results of a prospective multicenter clinical trial. J Clin Endocrinol Metab. 2014;99:1282-90.

5. Khan MS, El-Khouly F, Davies P, Toumpanakis C, Caplin ME. Long-term results of treatment of malignant carcinoid syndrome with prolonged release Lanreotide (Somatuline Autogel). Aliment Pharmacol Ther. 2011;34:235-42.

6. Oberg K, Lamberts SW. Somatostatin analogues in acromegaly and gastroenteropancreatic neuroendocrine tumours: past, present and future. Endocr Relat Cancer. 2016;23:R551-66.

7. Adelman DT, Burgess A, Davies PR. Evaluation of long-acting somatostatin analog injection devices by nurses: a quantitative study. Med Dev (Auckl). 2012;5:103-9.

8. Ryan P, Phan AT, Adelman DT, Iwasaki M. Neuroendocrine tumors and lanreotide depot: clinical considerations and nurse and patient preferences. Clin J Oncol Nurs. 2016;20:E139-46.

9. Adelman DT, Van Genechten D, Megret CM, Truong Thanh XMT, Hand P, Martin WA. Co-creation of a lanreotide autogel/depot syringe for the treatment of acromegaly and neuroendocrine tumours through collaborative human factor studies. Adv Ther. 2019;36:3409-23.

10. Novartis. Sandostatin ${ }^{\circledR}$ LAR summary of product characteristics. 2018. https://www.medicines.org. uk/emc/product/1038/smpc. Accessed 22 Nov 2019.

11. Ipsen Ltd. Somatuline ${ }^{\circledR}$ Autogel $^{\circledR}$ summary of product characteristics. 2019. https://www.medicines. org.uk/emc/product/8257/smpc. Accessed 22 Nov 2019.

12. Chen JG, Zou B, Shuster J. Relationship between patient satisfaction and physician characteristics. J Patient Exp. 2017;4:177-84.

13. Godden E, Paseka A, Gnida J, Inguanzo J. The impact of response rate on Hospital Consumer Assessment of Healthcare Providers and System (HCAHPS) dimension scores. Patient Exp J. 2019;6: 105-14. 
14. Indovina $\mathrm{K}$, Keniston A, Reid M, et al. Real-time patient experience surveys of hospitalized medical patients. J Hosp Med. 2016;11:251-6.

15. Reichheld F. The one number you need to grow: Harvard business review; 2003. https://hbr.org/2003/ 12/the-one-number-you-need-to-grow\#. Accessed 9 Jan 2019.

16. Salvatori R, Nachtigall LB, Cook DM, et al. Effectiveness of self- or partner-administration of an extended-release aqueous-gel formulation of lanreotide in lanreotide-naive patients with acromegaly. Pituitary. 2010;13:115-22.

17. Ryan P, McBride A, Ray D, et al. Lanreotide vs octreotide LAR for patients with advanced gastroenteropancreatic neuroendocrine tumors: an observational time and motion analysis. J Oncol Pharm Pract. 2019;25:1425-33.

18. Ström T, Kozlovacki G, Myrenfors P, Almquist M. Patient and nurse experience of using somatostatin analogues to treat gastroeneteropancreatic neuroendocrine tumors: results of the Somatostatin Treatment Experience Trial (STREET). Patient Prefer Adher. 2019;13:1799-807.
19. Alexopoulou O, Abrams P, Verhelst J, et al. Efficacy and tolerability of lanreotide Autogel therapy in acromegalic patients previously treated with octreotide LAR. Eur J Endocrinol. 2004;151:317-24.

20. Schiff M, Saunderson S, Mountian I, Hartley P. Chronic disease and self-injection: ethnographic investigations into the patient experience during treatment. Rheumatol Ther. 2017;4:445-63.

21. Matza LS, Stewart KD, Paczkowski R, Coyne KS, Currie B, Boye KSJJoP-RO. Psychometric evaluation of the Diabetes Injection Device Experience Questionnaire (DID-EQ) and Diabetes Injection Device Preference Questionnaire (DID-PQ). 2018;2:44.

22. Qin L, Chen S, Flood E, et al. Glucagon-like peptide1 receptor agonist treatment attributes important to injection-experienced patients with type 2 diabetes mellitus: a preference study in Germany and the United Kingdom. Diabetes Ther. 2017;8:335-53.

23. Pandya N, Losben N, Moore J. Optimizing insulin delivery for patients with diabetes. Geriatr Nurs. 2018;39:138-42. 